\title{
Morphological transitions in supercritical generalized percolation and moving interfaces in media with frozen randomness
}

\author{
Peter Grassberger (1) \\ JSC, FZ Jülich, D-52425 Jülich, Germany
}

(Received 15 May 2020; revised 12 August 2020; accepted 17 September 2020; published 28 October 2020)

\begin{abstract}
We consider the growth of clusters in disordered media at zero temperature, as exemplified by supercritical generalized percolation and by the $T=0$ random field Ising model. We show that the morphology of such clusters and of their surfaces can be of different types: They can be standard compact clusters with rough or smooth surfaces, but there exists also a completely different "spongy" phase. Clusters in the spongy phase are compact as far as the size-mass relation $M \sim R^{D}$ is concerned (with $D$ being the space dimension) but have an outer surface (or "hull") whose fractal dimension is also $D$ and which is indeed dense in the interior of the entire cluster. This behavior is found in all dimensions $D \geqslant 3$. Slightly supercritical clusters can be of either type in $D=3$, while they are always spongy in $D \geqslant 4$. Possible consequences for the applicability of Kardar-ParisiZhang (KPZ) scaling to interfaces in media with frozen pinning centers are studied in detail. In particular, we find-in contrast to KPZ-a weak-coupling phase in $2+1$ dimensions.
\end{abstract}

DOI: 10.1103/PhysRevResearch.2.043150

\section{INTRODUCTION}

Rough surfaces and interfaces have a huge number of applications in nature and in technology. Accordingly, there are many papers devoted to them in the physics literature, as well as several monographs [1-5]. For statistical physicists, one of the major reasons for studying them is the fact that they typically show anomalous (fractal) scaling, with deep connections to phenomena like phase transitions, critical points, and the renormalization group.

One very important distinction in the theory and phenomenology of rough surfaces is between growing and pinned ones. Pinned rough surfaces can only occur in media with frozen randomness at zero temperature, while moving rough surfaces can be found both in random media and in ordered media with temporal (thermal or athermal) disorder.

The prototypical model for driven interfaces without frozen disorder is the Kardar-Parisi-Zhang (KPZ) model [1], a discrete version of which is the Eden model for cancer growth [6]. When starting from a flat interface at time $t=0$, it never forgets the initial growth direction in any dimension, so that the interface never becomes isotropic either globally or locally (this is, e.g., different for diffusion limited aggregation, where interfaces become locally isotropic for $t \rightarrow \infty[7,8])$. Thus, growing interfaces in nonrandom media are self-affine.

This is not always the case for pinned interfaces. In two dimensions, it is shown in Refs. $[9,10]$ that critically pinned interfaces in isotropic random media are always in the univer-

Published by the American Physical Society under the terms of the Creative Commons Attribution 4.0 International license. Further distribution of this work must maintain attribution to the author(s) and the published article's title, journal citation, and DOI. sality class of critical percolation and thus fractal and isotropic on large scales. More precisely, the interfaces are in the class of percolation hulls, i.e., of externally accessible surfaces of critical percolation clusters. In $D \geqslant 3$, there are two different universality classes for critically pinned interfaces, depending on control parameters (plus a tricritical region for intermediate control parameters) [11-14]. One of them is again the percolation universality class, while the other is believed to be self-affine [2-4,12] — although there are numerical indications that this might not be strictly true, in particular, in the weak disorder limit [15]. The tricritical point separating these two regimes was studied using the renormalization group method in Ref. [13] and numerically in Ref. [14]. While the agreement between the two is far from perfect in $D=3$, at least the locations of the tricritical points are known with high precision for all $D<6$ [14].

In the present paper, we shall deal with the case of moving interfaces in (isotropic) media with frozen disorder. Notice that any frozen disorder leads, for sufficiently weak pushing, to pinning sites where the interface stops moving, while it continues to move globally. There is a widespread belief that they should also be described by KPZ scaling [2-5], because the "frozenness" of the noise should not be very relevant as long as the interface moves. It might occur occasionally that a particularly strong obstacle prevents the interface from progressing locally, in which case the interface will stop to grow locally and close again behind the obstacle. This leaves then a bubble, but these bubbles should not modify the basic scaling laws. Notice that most early experiments which looked for KPZ scaling in real phenomena [16-18] indeed involved frozen randomness.

We should stress that, as soon as (local) pinning is possible, the mapping of the KPZ problem onto the directed polymer model [19] breaks down, as the polymer would get adsorbed at the pinning center. Indeed, there is no theoretical argument 
why interfaces with local pinning should be in the KPZ universality class, although is is generally assumed [2-5].

An essential aspect of this standard scenario is that overhangs of the interface can be neglected in the scaling limit, i.e., the interface can be described by a single-valued height function $h(\mathbf{x})$. If there is no frozen randomness, i.e., in the $\mathrm{KPZ}$ case proper, this seems to be correct (although I am not aware of a rigorous proof). Detailed models of interfaces without overhangs [e.g., in so-called solid-on-solid (SOS) models] in the presence of frozen randomness have been studied in the literature [20,21], but the assumption that overhangs can indeed be neglected has, to my knowledge, never been challenged seriously.

As we shall see, it is indeed wrong. In some control parameter regions, this scenario is completely and qualitatively overthrown, due to the existence of what we call a "sponge phase," where the interface is even more rough than a fractal. In the sponge phase, the interface is indeed dense in the entire bulk underneath the surface, with deep fjords reaching from the top down to the bottom nearly everywhere. The standard scenario is still true qualitatively in other regions, but whether the KPZ scaling laws hold there is not completely clear. In order to study this, we have to define a suitable "effective interface," where the deep fjords in the sponge phase are cut off. Using this, we find clear evidence that there exists a "weak-coupling" phase in $D=3$ (i.e., $2+1$ ) dimensions, while such a phase exists for KPZ only for $D>3$ [22]. In the strong coupling phase, we find critical exponents roughly in agreement with KPZ.

In the next section, we shall define the model which we used for our numerics, but we stress that the phenomena discussed should hold much more generally. In Sec. III, we present theoretical arguments for what we call sponge phases and numerical results supporting them. In Sec. IV, we discuss whether KPZ scaling holds in nonspongy phases-and maybe even in sponge phases, provided one modifies suitably the definition of interfaces. In Sec. V, we summarize and draw our conclusions.

\section{GENERALIZED PERCOLATION}

We consider (hyper)cubic lattices in dimensions $D=3$ to 7. The class of models we study can be considered as a generalization of the susceptible-infected-removed (SIR) epidemic model, where we keep track of how often a (not yet infected) site had been "attacked" (and thus maybe weakened) by an infected neighbor [13]. Thus, each lattice site can be either removed (i.e., it had been infected, but it no longer is), infected, or in one of $\mathcal{N}+1$ susceptible states, where $\mathcal{N}$ is the coordination number. Time is discrete, and updating is done in parallel: At each time step, every infected site attacks each of its neighbors, after which it becomes removed. If a neighbor had already been attacked $k-1$ times (either previously or during the present time step) but is still susceptible, it becomes infected with probability $p_{k}$ [13]. Thus, after $k$ attacks it will have been infected with probability $q_{k}$ with

$$
q_{k}=q_{k-1}+\left(1-q_{k-1}\right) p_{k} .
$$

We shall call this model generalized percolation. For efficiency of the code, all infected (or "growth" or "active") sites are written in a list, and during each time step this list is gone through, a new list of growth sites is built, and at the end the old list is replaced by the new one. Notice that resulting configurations might depend on the order in which these lists are gone through, for a fixed sequence of random numbers. Therefore, after each time step the list of growth sites is randomly permuted.

Special cases of this model are site and bond percolation. In the former, a site cannot be infected at all if the first attack did not succeed. Thus, $p_{1}=p$ and $p_{k}=0$ for $k \geqslant 2$. For bond percolation, in contrast, $p_{k}$ is independent of $k: p_{k}=p$ for all $k$.

Consider now the random field Ising model (RFIM), with an initial state where all spins are down except for seed sites, whose spins are up. Dynamics is single flips with parallel update, and we assume that the spin at site $i$ can only flip if (i) this reduces the energy (i.e., we are at $T=0$ ) and (ii) at least one of it neighbors have flipped during the previous time step. It is easily seen $[9,10]$ that this can be mapped exactly onto the above generalized percolation model, for any distribution of local fields.

Alternatively, one could consider a porous medium that is wetted by a fluid with nonzero surface tension. Although one now cannot prove a general mapping, it is clear that generalized percolation will be a good model for a wide range of local geometries and surface tensions.

There are strong reasons [10-14] to believe that there are only three universality classes for critical generalized percolation. One is ordinary percolation. The second is found in cases where first attacks are very unlikely to succeed but lead to much weakened sites. Thus, $p_{k}$ increases strongly with $k$. In that case, clusters tend to be less fractal and their surfaces tend to be more smooth. Indeed, "bays" and "fjords" will become infected (because sites there have many infected neighbors), while tips and spikes are less likely to be formed. This is precisely the case for wetting of porous media by a fluid with high surface tension, and for the RFIM with very small randomness of the local fields. Indeed, it was found in Refs. [11,12] that there is a sharp morphological transition in critically pinned interfaces in porous media and in random magnets, where the interface changes from percolation-like at small surface tensions and strong disorder to rough but nonfractal at large surface tension and weak disorder. Between these two regimes is a tricritical point $[13,14]$. Essentially, we shall in the present paper deal with analogous (but very different in details) transitions for nonpinned interfaces.

In view of our claim of universality, we shall study only the simplest nontrivial model for generalized percolation, which was first studied in Ref. [14] and was called the minimal model (MM) in Ref. [10]. There, we assume $p_{1} \neq p_{2}$ but $p_{k}=$ $p_{2}$ for all $k>2$. Thus, we distinguish between virgin sites and sites that had already been attacked by an infected neighbor, but we do not keep track of the number of previous attacks.

In all dimensions, we follow the upward evolution of an interface that was initially equal to the (hyper)plane $z=0$, on lattices that were big enough so that the upper boundary of the lattice was never reached during the simulation. Lateral boundary conditions were helical. The critical lines in the $p_{1}$ versus $p_{2}$ control parameter space for $D=3,4$, and 5 are shown in Fig. 1. 

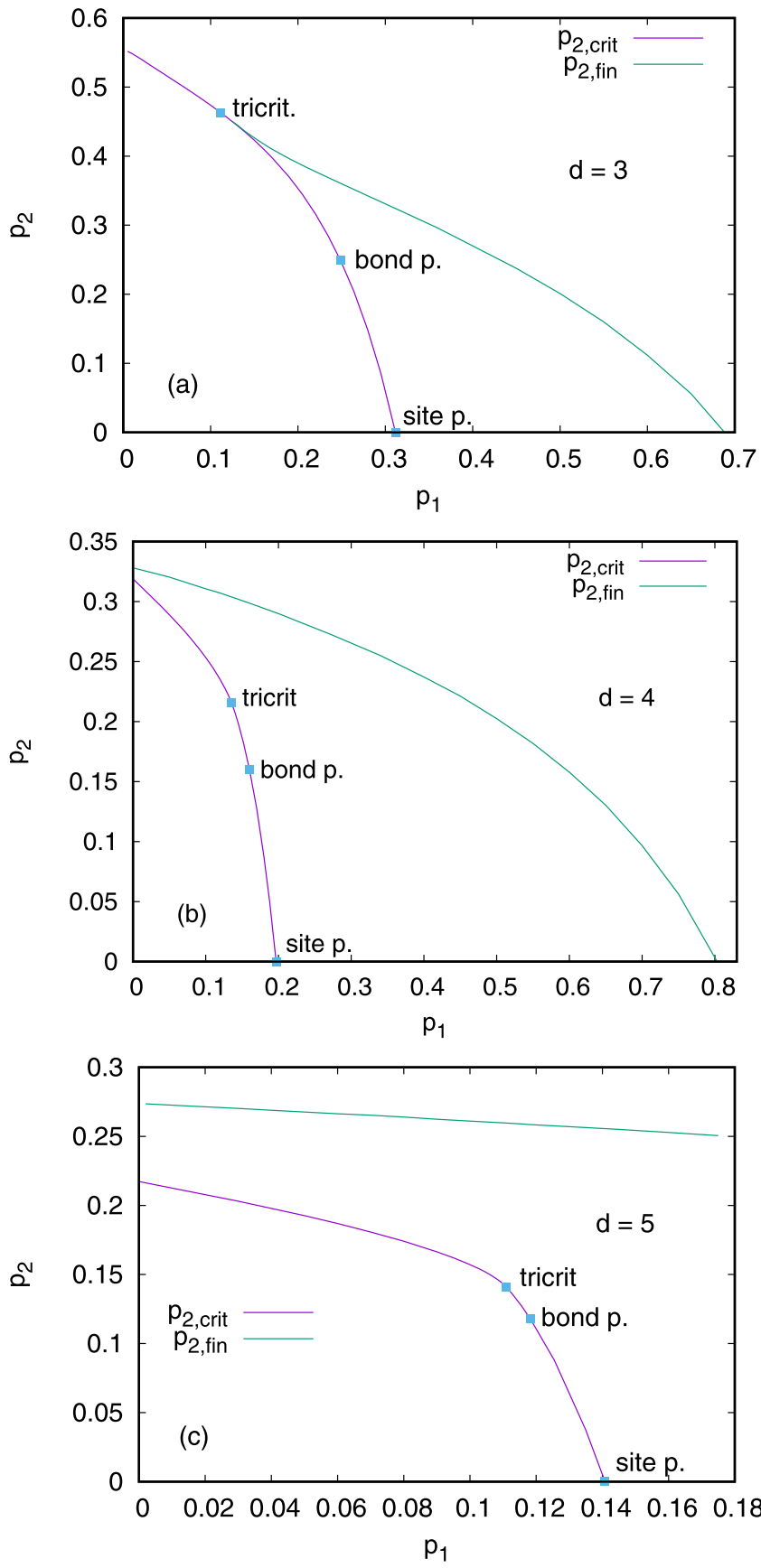

FIG. 1. Phase transition curves in dimensions 3 (a), 4 (b), and 5 (c). The lower (magenta) curves in each panel represent the critical curves (data from Ref. [14]). Below them, clusters starting from point seeds are finite and interfaces move only a finite distance before they stop. Above them, interfaces would grow forever on infinite lattices, and clusters starting from point seeds would have a finite probability to grow forever. On these critical curves are indicated the tricritical points [14] and the critical bond and site percolation points. Critical interfaces are fractal to the right of the tricritical points, while they are rough on their left sides. The upper (green) curves separate, for $D=4$ and 5 , the sponge phase (between both curves) from the nonspongy phase. For $D=3$, the same is true to the right of the tricritical point. On the left of the tricritical point, there is no sponge phase for $D=3$.

\section{MULTIPLE PERCOLATING CLUSTERS AND SPONGE PHASES}

Let us first consider site percolation in $D \geqslant 3$. For $D=$ 3 , the percolation threshold on the SC lattice is at $p_{c}=$ $0.3116 \ldots$ and for $D \geqslant 4$ it is even smaller [23]. Thus, $p_{c}$ in all these dimensions is less than $1 / 2$. Take now a hypercubic lattice and color its sites randomly black and white, each with probability $p=1 / 2$. Then both the black and the white sites will percolate; i.e., we have at least two coexisting percolating clusters. Indeed, since the density of black sites is supercritical, there will be exactly one infinite black cluster, and all other black clusters will be small. Similarly, there will be precisely one infinite white cluster. These two infinite clusters will then penetrate each other. If site $i$ is black and is on the infinite cluster, there is a finite probability that one of its neighbors is white and on the white infinite cluster. Finally, the same will be true not only for $p=1 / 2$, but also if sites are black with any probability $p \in\left(p_{c}, 1-p_{c}\right)$ and white with probability $1-p$.

Consider now a cluster of black sites grown - for a finite time $t$, and for $p \in\left(p_{c}, 1-p_{c}\right)$-by starting from an infected hyperplane $z=0$ that can infect its upper neighbors [24]. Otherwise, consider the set of all black sites (in a configuration with a fraction $p$ of black sites) which are connected to the bottom of the lattice by a path of length $\leqslant t$. Roughly, they will occupy densely a layer of thickness $\propto t$. Similarly, when starting from a point seed, the cluster will occupy densely a (hyper)sphere of radius $\propto t$. By "densely," we mean that this layer or sphere has no big voids (near every point in the layer or sphere there is a point belonging to the cluster), but it contains many holes, and these holes are not only connected but also dense. Indeed, the white infinite cluster will also be dense everywhere, so that the black cluster has fjords that penetrate it all the way down to the bottom $z=0$.

We call the phase with two clusters penetrating each other and being dense everywhere a sponge phase. While the above arguments clearly show (even if not in a strict mathematical sense) that a sponge phase exists in site percolation for $D>3$, the situation is much more subtle for bond percolation. The existence of multiple clusters was proven rigorously at least for $D \geqslant 8$ by Bock et al. [25], and their denseness was proven in Ref. [26].

In order to understand the situation for bond percolation with all $D \geqslant 3$ and for generalized percolation, we used Monte Carlo simulations. We studied mainly the planar geometry. Thus, in a first step, we started with a seed consisting of the hyperplane $z=0$ and let the cluster grow until the layer $z=L_{z}-1$ was reached for the first time. At that moment, the growth was stopped, and in a second step a cluster was grown on the not yet infected sites by starting from a (hyper)planar seed at $z=L_{z}$ and moving down. Alternatively, in order to speed up the simulation, we did not grow the entire second cluster but just followed the external hull of the first cluster (by which we mean the set of sites which are not on the first cluster but are neighboring it and which are accessible by paths starting at $z=L_{z}$ and avoiding the first cluster).

As observables, we measured the penetration depth of the second cluster, its density profile, the mass of the hull, and its variance. In general, the lateral size $L$ of the lattice was somewhat smaller than $L_{z}$. 

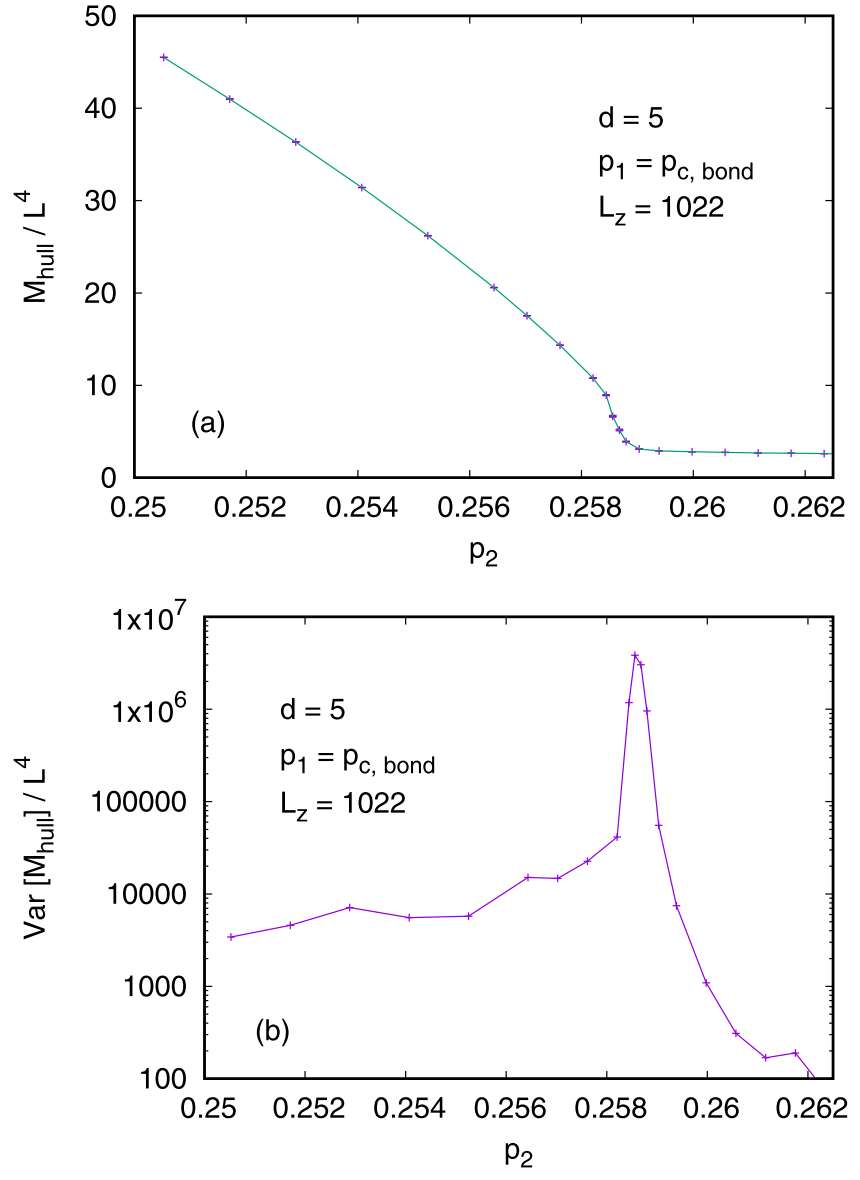

FIG. 2. (a) Mass density (per unit base surface) of the external hull of the cluster growing upwards from $z=0$ in dimension $D=$

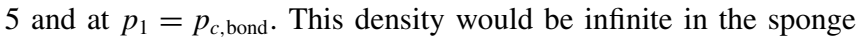
phase, if the cluster thickness $L_{z}$ were infinite. Panel (b) shows the variance of the mass density.

As typical results, we show in Fig. 2(a) the average mass density of the hull and in Fig. 2(b) its variance, for $D=5$ and for $p_{1}=p_{c \text {, bond }}$. The mass density is defined per unit area of the base surface. Both plots show very clearly a phase transition at $p_{2}=p_{2, \text { sponge }}=0.2586(5)$. Notice that $p_{c \text {, bond }}=$ 0.11817 for $D=5$, i.e., there is a rather wide region with a sponge phase. For $p_{2}>0.2586$, the first cluster is so dense that the second one cannot penetrate and there is no sponge phase.

Similar plots were made for many more values of $p_{1}$ and for all dimensions between 3 and 7 . Final results for $D \leqslant 5$ are plotted in Fig. 1 together with the curves for the percolation (or pinning) transitions.

Notice that it would not be easy to obtain the order of the sponge transition or any critical exponents from plots like Fig. 2. Partly this is because these plots depend strongly on $L_{z}$. For $L_{z} \rightarrow \infty$, not only the variance but also the average mass would diverge. But fortunately, the transition can be studied much more precisely by another type of simulation.

There we use a lattice of size $L^{D}$ with periodic boundary conditions [27]. In a first step, we determine the percolating cluster, if there is one. Since we do this deep in the supercritical region, this is practically always the case, and its

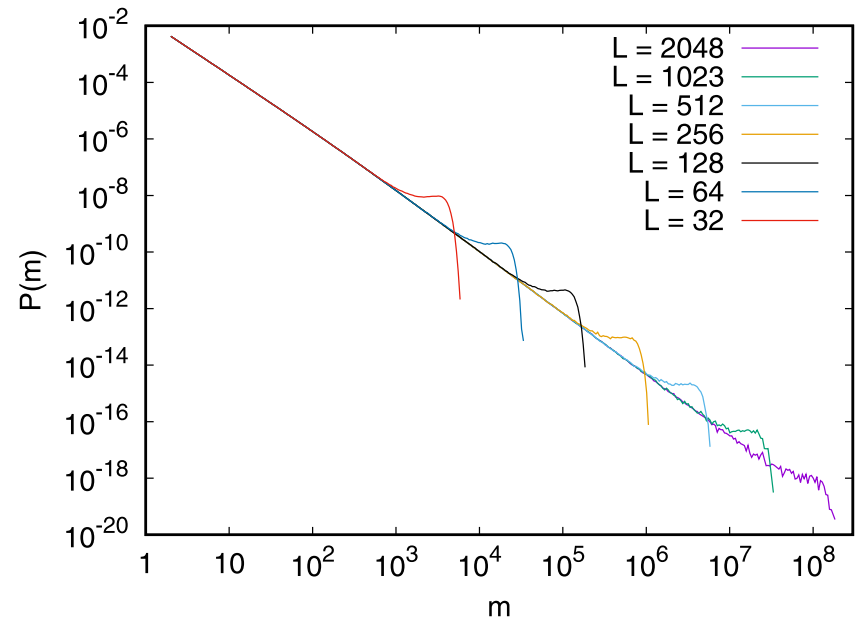

FIG. 3. Mass distribution of clusters inside the holes of the giant cluster in supercritical bond percolation in $D=3$, with $p=$ $p_{b, \text { sponge }}=0.31958$ chosen such that the holes just contain a incident giant cluster (notice that this second cluster is grown with $p=1$, i.e., we are at the threshold where a giant cluster exists in the complement of the first cluster). As in ordinary 3-D percolation, we have $P(m) \sim m^{-\tau}$ with $\tau=2.189$ in the scaling region and a peak position scaling $m_{\max } \sim L^{D_{f}}$ with $D_{f}=2.523$.

determination is easy. In a second step, we look for a percolating cluster with $p_{1}=p_{2}=1$ on its complement. Notice that we cannot show in this way whether the second cluster penetrates densely the first one, but we can determine the transition point with higher precision and we can study the second cluster more carefully near the transition. A typical result is shown in Fig. 3, where we plotted the mass distributions of all clusters in the holes of the first (supercritical) cluster for bond percolation at $p=p_{b \text {,sponge }}$ in $D=3$. They have the standard Fisher exponent $\tau=2.189$ and the fractal dimension $D_{f}=2.523$ of ordinary percolation. Similar plots for dimensions $D=4$ to 7 show that the problem is always in the ordinary percolation universality class. This might not be obvious in view of the fact that percolation inside holes of critical percolation clusters in $D=2$ is in a different universality class [28]. But it actually is not very surprising, since the first cluster-being strongly supercritical - is very similar to the set of all black sites, and thus the second largest (white) cluster grows on a very weakly correlated set of randomly chosen sites. This is very different in $D=2$. A list of sponge transitions for bond percolation is given in Table I. These

TABLE I. Sponge transition points for bond percolation in dimensions $D=3$ to 7 . In the last column, we give the thresholds for directed percolation on hypercubic lattices with the diagonal as preferred direction [29].

\begin{tabular}{lcc}
\hline \hline$D$ & $p_{b, \text { sponge }}$ & $p_{b, \text { dirperc }}$ \\
\hline 3 & $0.31958(1)$ & 0.38222 \\
4 & $0.27289(1)$ & 0.26836 \\
5 & $0.24081(1)$ & 0.20792 \\
6 & $0.21582(1)$ & 0.17062 \\
7 & $0.19671(2)$ & 0.14509 \\
\hline \hline
\end{tabular}


values should be compared to the asymptotic estimate

$$
p_{b, \text { sponge }} \sim \log (D) / 2 D
$$

for large $D$ [25].

As seen from Fig. 1, there is a striking qualitative difference between $D=3$ and $D \geqslant 4$ : While there is a spongy phase for all values of $p_{1}$ when $D \geqslant 4$, no such phase exists

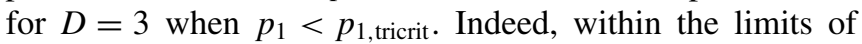
accuracy, the spongy phase seems to exist precisely down to $p_{1}=p_{1 \text {,tricrit }}$. We have no theoretical explanation for this. It means that in $D=3$ the depinning transition always leads to a nonspongy growing cluster when the pinned interface is nonfractal but always leads to a spongy growing cluster when it is percolation-like. There is no such distinction for $D \geqslant 4$.

\section{ARE GROWING INTERFACES IN MEDIA WITH FROZEN PINNING CENTERS IN THE KPZ UNIVERSALITY CLASS?}

\section{A. General remarks and a second morphological transition related to directed percolation}

When there is a sponge phase, the answer to the above question is, at least in a naive sense, "no." The true interface is in this case extremely convoluted, has fractal dimension $D_{f}=D$, and penetrates densely the entire bulk phase underneath the surface. One can presumably define then an outer interface where all fjords are cut off, in which case one would follow what would look like the interface in a coarse-grained sense. Then we still have several obvious questions:

(1) If we are not in a spongy phase, is the interface scaling described by a single universality class or are there different universality classes?

(2) Is at least one of these the KPZ universality class?

(3) If there are several classes, are there further morphological transitions between them?

(4) Does there exist a "natural" definition of an effective interface in the spongy phase where fjords are (at least partially) cut off, and does KPZ scaling hold for it?

A partial answer is suggested by the fact that the KPZ equation has weak and strong coupling solutions, but only for $D>3[22,30]$. The strong coupling solution is the one that shows the standard KPZ scaling, while interfaces in the weak coupling regime are asymptotically flat with logarithmic corrections; i.e., the interface width increases less quickly than any power of the base length $L$ in the limit $t \rightarrow \infty$. A similar weak to strong coupling transition might occur in our model already for $D=3$. Indeed, when $p_{1}$ is very small, the growth at tips of the interface is very strongly suppressed (maybe stronger than by any finite diffusion constant in the KPZ equation), and the interface can grow only if bays are filled up sufficiently quickly-leading thereby to a nonrough interface asymptotically.

Another partial answer is also suggested by the fact that yet another morphological transition for interface growth with bounded speed of spreading is provided by the threshold of directed percolation (DP) [30-32]. Consider, more precisely, bond percolation from an initial wetted surface in $D \geqslant 3$ with Miller indices $(1, \ldots, 1)$, such that growth is along the space diagonal. Denote by $p_{c}$, bond the bond percolation threshold in $D$, and by $p_{b \text {,dirperc }}$ the threshold for directed bond percola- tion on a $D$-dimensional hypercubic lattice with growth in the $(1, \ldots, 1)$ direction. As long as $p_{c \text {,bond }}<p<p_{b \text {, dirperc }}$, a typical shortest path to a wetted site with $z \gg 1$ will have many back turns, simply because long paths without back turns would not exist for $p<p_{b \text {,dirperc }}$. This is, however, no longer true for $p>p_{b \text {,dirperc. In this regime, many such paths }}$ exist, and there is a nonzero probability that a site is wetted by such a path. Thus, there is a finite probability that sites with $z=t$ get wetted at time $t$, and the active sites are on a perfectly flat surface-although the latter has fjords and holes penetrating it nearly everywhere. If we would cut off these fjords by some ad hoc rule, we would obtain a flat interface.

This last argument rests on the assumption that we have a regular lattice with frozen percolative disorder. It would no longer hold if we had in addition annealed disorder (e.g., with random times needed to jump over a lattice bond) or if the frozen disorder were of a different type. In that case, there would still be a directed percolation transition at which back-bending paths become irrelevant, but paths without back-bending would not arrive at the same hight at any given time. Thus, the interface would still be rough, and the standard arguments for KPZ scaling in cases with annealed disorder (in particular the relationship with directed polymers $[19,30])$ would suggest that such interfaces are in the KPZ universality class.

Thus, we should not expect the DP-related morphology transition to be universal, while we do expect the sponge transition to be universal. Nevertheless, it is of interest to look at the numerics. Critical vales for DP on hypercubic lattices with spreading direction along the diagonal [29] are given in

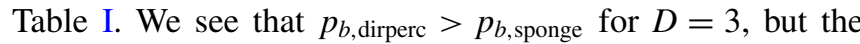
opposite is true for $D>3$. Thus, we have no regime where we can expect true KPZ scaling of supercritical bond percolation interfaces in dimensions $D \geqslant 4$; there is a possible window ( $p \in[0.3196,0.3822]$ for bond percolation) for $D=3$. Notice, however, that we can still have KPZ scaling for effective interfaces in higher dimensions, a question which we will not study in the present paper.

In the following, we shall study only the case $D=3$, in a wide parameter range. These will include spongy and nonspongy phases, and weak and strong coupling. Although our results are affected by very large finite size corrections, we shall argue that a weak coupling phase exists and that KPZ scaling holds at least approximately in the strong coupling regime for effective interfaces-even in the spongy phase.

\section{B. Supercritical interfaces in $D=3$}

In this subsection, we show simulations for the minimal model in $D=3$. We use plane seeds oriented in the $(1,1,1)$ direction, i.e., with the normal to the plane parallel to the space diagonal of the lattice. This model bears some resemblance to the cube-stacking model of interface growth [33], but with three important differences:

(1) The cube-stacking model is not a percolation model but of Eden [6] type; i.e., there are no dead (or 'removed') sites, and growth at any site can occur at any time, although it had stopped temporarily before.

(2) In the cube-stacking model, the interface can also recede locally, by cubes detaching from the interface. 
(3) In the cube-stacking model, sites can be wetted only if they have three wetted neighbors, while we allow growth already for one or two wetted neighbors. This can create holes and overhangs, which are not possible in the cube-stacking model.

With such an orientation, interfaces can grow even if $p_{1}=$ 0 , i.e., even if two neighbors are needed to wet a site. Lateral boundary conditions are helical, but for most efficient use of the memory we used tilted coordinates $i$ for the horizontal and $z$ for the vertical position, such that the neighbors of site $(i, z)$ are $(i, z+1),(i, z-1),(i+1, z+1),(i-1, z-$ $1),(i+L, z+1),(i-L, z-1)$. If we had used the original tilted coordinates, every second memory location would be unused.

In the $z$ direction, lattices were de facto infinitely large, because we used a recycling trick: First, we determined in test runs the roughness in worst cases. Assume that we estimated that the width-for the given lateral size $\mathrm{L}$ and for the given values of $p_{1}$ and $p_{2}$-is less than $W_{\max }$ with high probability. We introduced a height variable $z_{0}$ and initiated it as $z_{0}=0$. Then, if the highest site in the interface had reached a height $z_{\max }>W_{\max }+z_{0}$, we erased the plane $z=z_{0}$, increased $z_{0}$ by 1 unit, and used the erased plane to store the parts of the interface with $z=z_{\max }$. During these simulations, we checked that $W$ indeed remained bounded by $W_{\max }$. If this was violated, we discarded the entire simulation and repeated it with a higher value of $W_{\max }$

As a last trick to use memory most efficiently, we used multispin coding. The system sizes that we could handle in this way depend on the interface roughness and thus also on the distance from the critical percolation line. For small roughnesses, i.e., far above the percolation threshold, we could simulate systems with base surfaces up to $L \times L=$ $8192 \times 8192$ and followed them typically for $2 \times 10^{5}$ time steps (for smaller base surfaces, up to $L=2048$, we went up to $10^{6}$ to $10^{7}$ time steps). This is to be compared to the largest previous simulation of interfaces with overhangs (in the RFIM), where $t \approx L \leqslant 250$ [34].

\section{Interface width scaling for $t \rightarrow \infty$}

For small times, KPZ scaling is masked by the local roughness of the interfaces, and studying it numerically requires particular care. We postpone this to the next subsubsection and present here only data for large times, when the interface moves with constant average speed and its statistical properties are stationary. Since our interfaces had overhangs and the bulk phase below had holes, the definition of an interface height is not unambiguous. For simplicity, we show results where the average height and its variance at time $t$ are given by the average height and variance of the active (i.e., newly wetted) sites. We verified, however, that similar results were also obtained by other definitions, e.g., if the (effective) interface height at horizontal position $i$ is given by the highest wetted site in the column $\{(i, z) ; z \geqslant 0\}$.

For KPZ in three dimensions, the width of a moving interface in the stationary state scales as

$$
W(L) \sim L^{\alpha}
$$

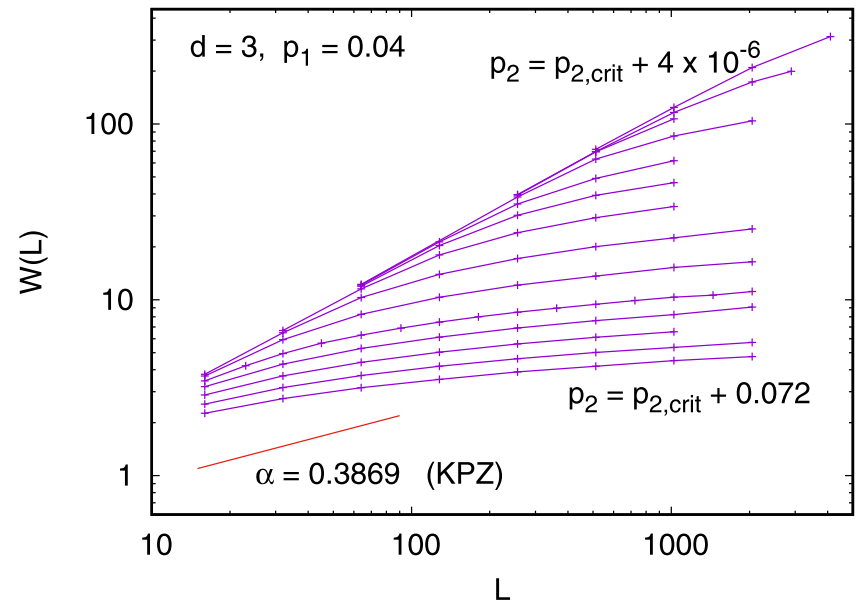

FIG. 4. Interface widths in the stationary state for large times vs base size $L$. The short red straight line represents the KPZ scaling $W \sim L^{\alpha}$ with $\alpha=0.3869$. Each of the other curves is for one value of $p_{2}$, with $p_{1}=0.04$ being common to all of them. The lowest curve is the one for largest $p_{2}$, while the uppermost curve is for $p_{2}$ just slightly above the percolation threshold, i.e., for interfaces that are nearly pinned. More precisely, the values of $p_{2}$ are (from top to bottom) $0.52432,0.52436,0.5244,0.52452,0.5248,0.5252,0.526,0.528$,

$0.532,0.54,0.548,0.56,0.576$, and 0.596 .

with $\alpha=0.3869 \pm 4$ [35]. In Fig. 4, we compare this (short straight line) to data for $p_{1}=0.04$. Each curve here corresponds to one value of $p_{2}$. The uppermost curves essentially show the scaling of nearly pinned interfaces. The upper envelope gives $W \sim L^{0.84}$ for just marginally unpinned interfaces. All curves are convex. If this convexity prevails also for larger $L$, each curve gives an upper bound for $\alpha$. Indeed, the slopes of all curves seem to become the same, giving the bound $\alpha \leqslant$ $0.10(1)$, in striking disagreement with the KPZ prediction.

In order to see whether this depends on the particular value of $p_{1}$, we show in Fig. 5 analogous results for $p_{1}=0$. We plot the data differently there to indicate also the scaling for small $p_{2}-p_{2, \text { crit }}$, where $p_{2, \text { crit }}=0.559188$ is the pinning threshold.

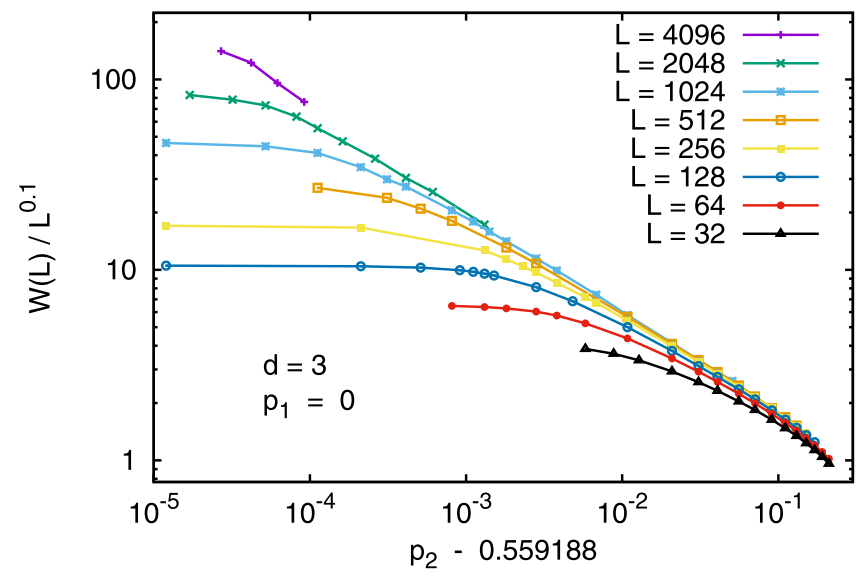

FIG. 5. Interface widths in the stationary state for large times at $p_{1}=0$ vs $p_{2}-p_{2, \text { crit }}$. Each curve corresponds to one fixed value of $L$. Anticipating that $W(L) \sim L^{\alpha}$ with $\alpha \leqslant 0.1$ for large $L$, we actually plotted $W(L) / L^{0.1}$ 


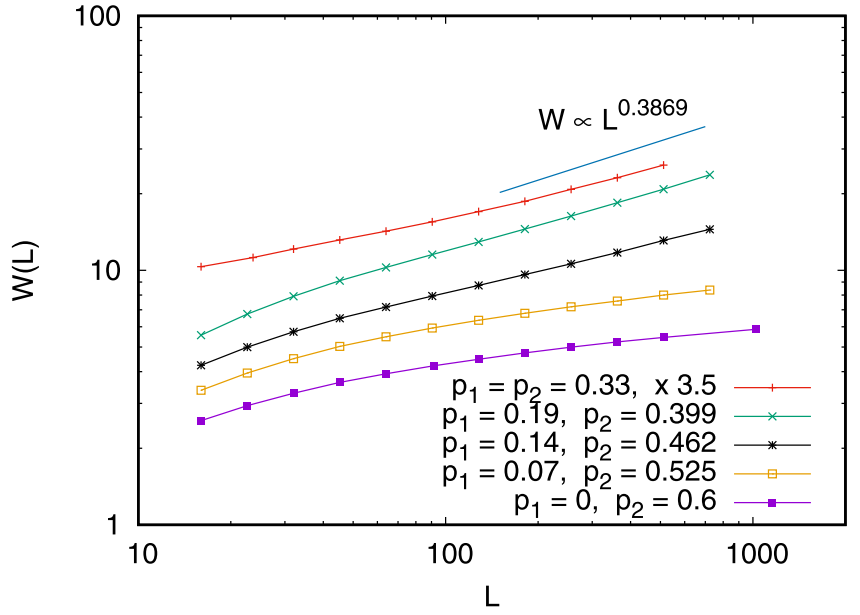

FIG. 6. Interface widths in the stationary state for large times at five pairs $\left(p_{1}, p_{2}\right)$ vs base size. The curve for bond percolation $\left(p_{1}=\right.$ $p_{2}$ ) is shifted up to avoid crowding of the curves. The short straight line indicates again KPZ scaling.

More precisely, we plotted in Fig. 5 the ratios $W(L) / L^{0.1}$, anticipating that $W(L)$ scales also for $p_{1}=0$ with a similarly small exponent $\alpha$ as for $p_{1}=0.04$. This seems to be indeed the case, and again our results are in striking disagreement with the KPZ prediction.

These results might suggest that there is indeed another nontrivial power law, in addition to the KPZ scaling and to the trivial scaling $W=$ const of the weak-noise solution of the KPZ equation. We consider this, however, as extremely unlikely and interpret the data in Figs. 4 and 5 as logarithmic growth of $W(L)$, as is indeed expected for a weak coupling phase.

Results for larger values of $p_{1}$ are shown in Fig. 6. They are all for values of $p_{2}$ which are slightly $(\approx 5 \%)$ higher than the critical ones, except for those for bond percolation. There, $p$ had to be substantially larger than $p_{c}$ because we wanted it to be outside the sponge phase. To avoid crowding of curves, we thus shifted the curve for bond percolation by multiplying $W$ by a factor 3.5. We see that curves for $p_{1}<0.1$ bend down for all $L$, while curves for $p_{1}>0.1$ veer up for large $L$. Although none of the curves reaches the KPZ scaling at large $L$ (for this we would need much larger system sizes), we interpret this as an indication that the transition between weak and strong coupling happens near $p_{1} \approx 0.1$. This is also supported by Fig. 7 , where we show again the $p_{1}$ versus $p_{2}$ plane and indicate by bullets (crosses) points in the weak (strong) coupling regime. All this suggests a weak to strong transition near the short line in Fig. 7. It is very tempting to suggest that this line meets the critical line precisely at the tricritical point. This fascinating conjecture would mean that four different transition lines meet at this point: The critical curve for self-affine pinned surfaces, the critical percolation curve, the sponge transition, and the weak to strong KPZ transition. It would also explain why the very careful RG study of Janssen et al. [13] disagreed so dramatically with the numerics of Ref. [14]. At its upper end, the weak to strong transition curve ends on the critical DP curve; more precisely it seems to end at the tricritical DP point. The latter is identified by the tricritical scaling for the

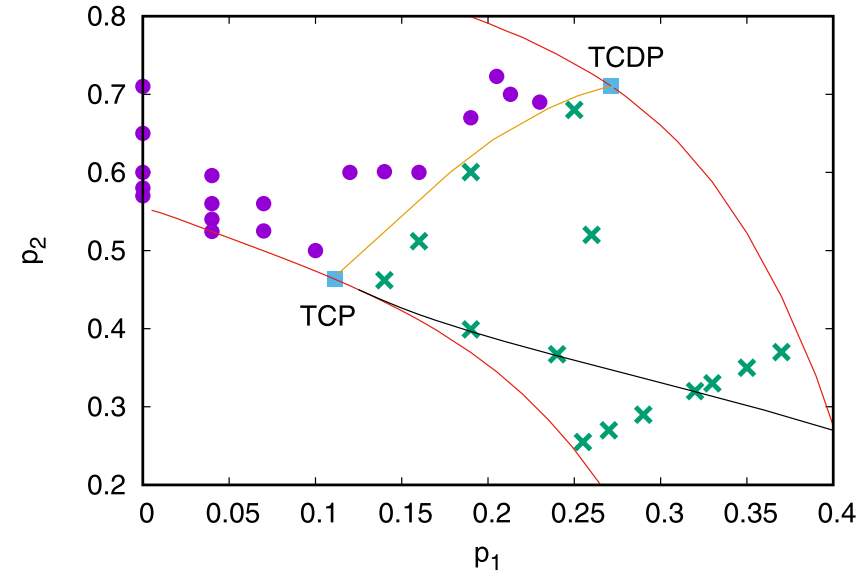

FIG. 7. Phase diagram for $D=3$ with weak coupling KPZ points indicated as bullets and strong coupling points indicated as crosses. The weak vs strong classification is done on the basis of the curvatures of $W(L)$ vs $L$ curves (such as those in Fig. 6) for $L>128$. The uppermost (red) curve indicates critical DP, with tricritical DP marked (like tricritical percolation as well) by a square (light blue). The curve running bottom left to top right (dark yellow in online version) is a rough estimate of the weak vs strong transition curve. It ends on both sides at tricritical points.

density of wetted sites, $\rho(t) \sim t^{-0.087 \pm 0.003}$ and the survival probability of a point seed cluster, $P(t) \sim t^{-1.218 \pm 0.007}[36]$ [which gives $\left.\left(p_{1}, p_{2}\right)_{\mathrm{TCDP}}=(0.2709(3), 0.7111(5))\right]$.

In the strong coupling regime, there are (as in the regime with weak coupling) huge corrections to scaling, which makes a precise determination of $\alpha$ very difficult. The best estimates, resulting from the points $\left(p_{1}, p_{2}\right)=$ $(0.26,0.52),(0.24,0.3672),(0.32,0.32)$, and $(0.33,0.33)$, are $\alpha=0.37(2)$. This suggests that the strong coupling regime is in the KPZ universality class, although there is also room for caveats.

\section{Time dependence of interface widths}

Typical results for bond percolation at $p=0.32$, i.e., in the strong coupling region slightly above the sponge transition, are shown in Fig. 8. We see essentially three regimes:

(1) For very short times $(t<10$ in the present case), the widths are dominated by the fuzziness of (near-critical) percolation. In this regime, there is no dependence on $L$, and - as we shall see later-also no dependence on $p$.

(2) In the intermediate time regime $\left(10<t<10^{3}\right.$ in the present case), the "microscopic" (percolative) roughness is overtaken by the KPZ-type roughness, but times are still so small that the considered lattices are effectively infinite. Thus, there is still no dependence on $L$, but—as we shall see laterthere is dependence on $p$.

(3) Finally, there is the large- $t$ regime where the width no longer can grow. Rather, interfaces are there (statistically) stationary. This is the regime studied in the previous subsubsection.

In Fig. 8, we included also a curve for $L=8192$, for which the stationary regime is not yet seen. We did this in order to make clear that the curves are not simply straight lines in the 


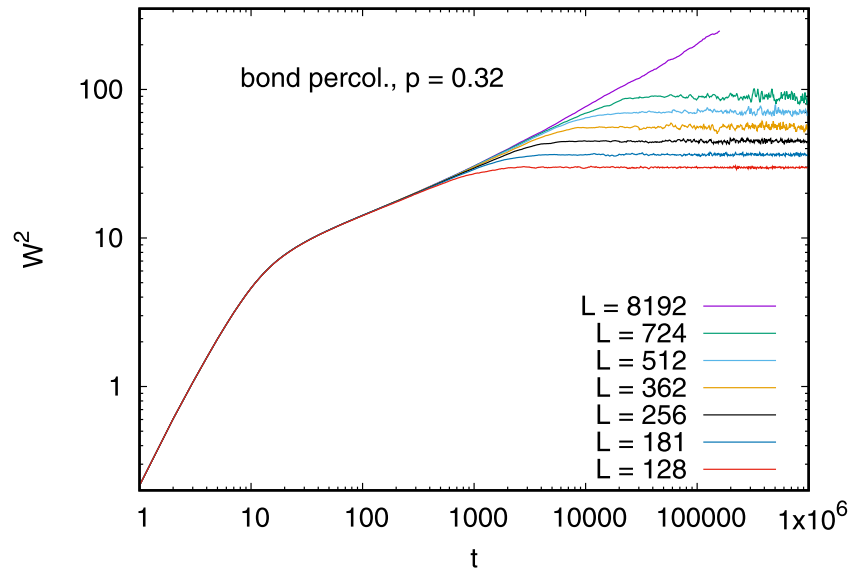

FIG. 8. Log-log plot of squares of effective interface widths for bond percolation at $p=0.32$. Each curve corresponds to one value $L$ of the system size, and the heights of the curves increase with $L$.

intermediate regime. This will become even more obvious in the next two figures.

In Fig. 9, we show again $W^{2}$ versus $t$ (again for bond percolation) but now we keep $L=8192$ fixed, and vary $p$. Also, due to the very large $L$ all data in Fig. 9 are in the smalland intermediate- $t$ regimes. We compare these data with two power laws indicated by straight lines. The straight line on the left indicates the behavior for critical percolation. It does not seem to fit perfectly, but this is (in spite of the large value of $L)$ a finite-size effect. Closer scrutiny shows that the data are there in perfect agreement with theory. The straight line on the right-hand side corresponds to what is expected for KPZ scaling. There the agreement is not as good. For the times shown in this figure, all curves are more flat than predicted for $\mathrm{KPZ}$, but this is maybe due to the curvature seen already in the

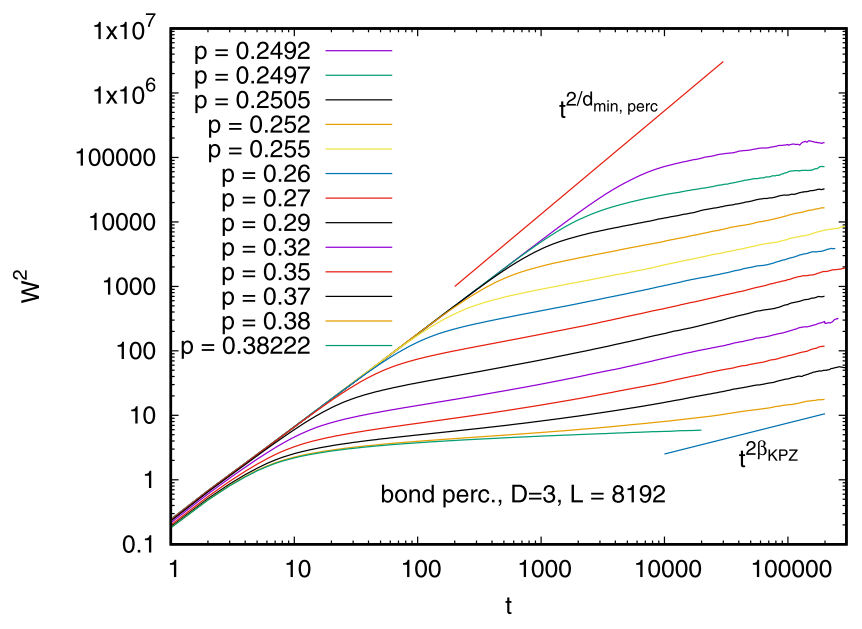

FIG. 9. Log-log plot of squares of effective interface widths for bond percolation at $L=8192$ and different values of $p$. Notice that all values of $p$ are supercritical, but some are in the sponge and others are in the nonsponge phases. The lowest curve (with the largest value of $p$ ) is for the DP transition, while the uppermost curve is just barely supercritical. Nothing special is seen near the sponge transition, which is at $p \approx 0.32$. The two straight lines are the scalings expected for $p=p_{b, \text { crit }}$ and for KPZ.

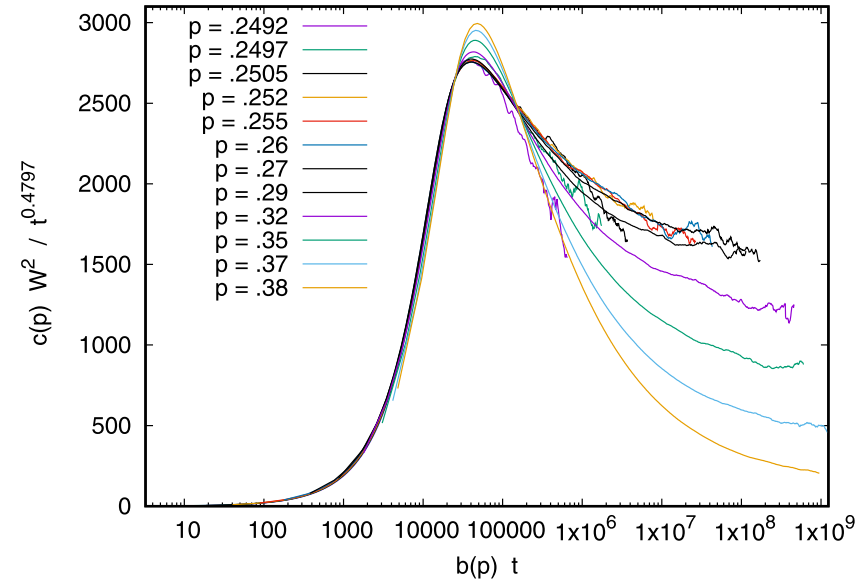

FIG. 10. Same data as in Fig. 9, but curves are shifted horizontally and vertically, and divided by $t^{2 \beta_{\mathrm{KPZ}}}$. Plotted in this way it becomes obvious that systems are too small for $p<0.251$ to show a possible KPZ scaling, while $t$ is too small for $p>0.28$.

previous figure. We cannot rule out that the curves would for larger $t$ (and thus also for larger $L$ ) slowly approach the KPZ prediction. To make this even more clear, we plotted the same data in a slightly different way in Fig. 10, where we multiplied each curve by $t^{-2 \beta_{\mathrm{KPZ}}}$ and shifted it horizontally and vertically, in order to enhance the significance of the plot.

We should stress that Figs. 9 and 10 also include data from the sponge phase, where the true interface width would increase linearly with $t$. What these data show is that our definition of effective interfaces cuts off fjords sufficiently, so that they have even a good chance to satisfy KPZ scaling.

Finally, we should mention that data in the weak coupling regime look qualitatively similar to Figs. 8 and 9, with three regimes dominated by internal roughness (for small $t$ ), by logarithmic increase of $W$ (intermediate $t$ ) and corresponding to asymptotic stationarity. We do not show these data because they no not look very distinct.

\section{DISCUSSION AND CONCLUSIONS}

The main result of the present paper is the establishment of a sponge phase. Similar phases with two tightly intermingled microscopic phases were previously known only for systems with at least three microscopic phases, e.g., in emulsions with two liquids and one membrane [37]. In the present case, there are no explicit membranes between the two microphases, which are made up of interpenetrating supercritical percolation clusters. The striking property which distinguishes a sponge phase from other multiphase systems is that both microphases are connected and everywhere dense in the mathematical sense, i.e., in the scaling limit every point in space is infinitely close to points in both microphases.

This sponge phase implies that the standard scenario for interfaces moving in locally isotropic media with quenched randomness, going back to a famous paper by Bruinsma and Aeppli in 1984 [20], is wrong. It basically says that such interfaces become more smooth as dimension increases. While this is correct for strongly pushed interfaces far from local pinning, the opposite is true close to the pinning transition: 
There, moving interfaces become more fuzzy with increasing dimension. What is however still true - at least for $D=3$-is that sites where the interface moves are located on a smooth manifold, even when the interface itself is not smooth.

For $D>3$, we found that the transition from pinned to moving interfaces in media with frozen pinning centers always leads first to a sponge phase, while nonspongy bulk phases appear only in a second morphological transition. This is at least what we found in what was called the "minimal model" in Ref. [10], but we conjecture that the same is true also for the RFIM with any other distribution of local random fields.

For $D=2$ no sponge phases can exist (for topological reasons), and for $D=3$ we found that the depinning transition can lead either to a sponge or to a nonsponge phase. The first seems to happen when the depinning transition is in the percolation universality class, while the latter happens when the critically pinned interface is self-affine. Again, this claim is only based on the minimal model. Simulations with other models in the generalized percolation family (which includes the zero- $T$ RFIM) would be extremely welcome to verify it, as would be analytic arguments. So far, these claims are entirely based on simulations.

At the sponge to nonsponge transition, the incipient infinite clusters in the voids of the main phase are in the universality class of critical ordinary percolation. Again we claim that this is true in general, although we have only simulation data to support it.

In the last part of the paper, we asked whether the interfaces in the parameter region above the sponge phase satisfy KPZ scaling, as is often assumed-in spite of the fact that theoretical arguments for KPZ scaling only exist in cases without frozen pinning centers. Moreover, we asked whether suitably defined effective interfaces could satisfy KPZ scaling even in the sponge phase. Here we studied only the case $D=3$. We found huge finite-size corrections, but our simulations were nevertheless able to support the following scenario: For all control parameters, the interfaces are asymptotically either in a strong coupling universality class or in the weak coupling class. In the latter, the interface width increases, for large times, less quickly that any power of the base length $L$, so that it becomes asymptotically flat.

Whether the strong coupling universality class is really identical with the KPZ class is still an open problem. Usually it is taken for granted, but without any compelling theoretical or numerical arguments. In all present simulations, corrections to scaling were extremely large, so that no firm conclusion could be drawn, but the values of the exponents $\alpha$ and $\beta$ found in the strong coupling domain suggest that moving rough interfaces in media with frozen pinning centers might indeed be in the KPZ class.

Marginally supercritical (i.e., nearly pinned) interfaces in $D=3$ seem to be rough only when the critically pinned interfaces are percolation hulls in the standard percolation class, while depinning via self-affine critical interfaces leads to asymptotically flat interfaces (weak coupling KPZ phase). This might not be the most important finding of the present paper, but it certainly is the most surprising - together with the observation that the weak to strong coupling transition curve ends also at its other end at a tricritical point, namely that of
DP. The fact that the weak to strong coupling transition curve ends on both sides at tricritical points suggests strongly that this is not merely a numerical accident.

To many readers, the very existence of a weak coupling (i.e., nonrough) phase in $D=3$ will be the most surprising result. It presents a clear violation of the often assumed KPZ universality. Reasons for possible nonuniversality have been discussed in the literature [38-43], but none of these seems to apply in the present case. They include unusual noise distributions in high dimensions [38], pulled fronts [39], anisotropies in the KPZ nonlinearities [40], and vanishing of the KPZ nonlinearity parameter [41-43].

Although our noise looks unconventional in the RFIM interpretation, it is very similar to a simple Gaussian [10], and according to Ref. [38] we should expect problems anyhow only for $D>3$. In the terminology of Ref. [39], our fronts look much more like pushed than like pulled fronts, in particular for small $p_{1}$, where we see the weak coupling phase. Our model is anisotropic in the sense that the speed of propagation of a tilted interface depends on the orientation of the tilt, but it has a sixfold symmetry in the sense that the speed is lowest when the tilt makes the interface close to a coordinate plane, while it is maximal when it is in the direction of one of the six diagonals. In contrast, an effect was found in Ref. [40] only for twofold symmetries. Finally, there is no indication in our model that the KPZ nonlinearity vanishes, in contrast to the model of Ref. [41]. Also, in the latter the weak coupling phase is, strictly speaking, observed only at an isolated parameter value, while we observe a true phase in an extended parameter region.

In contrast to these effects, we believe that the origin of the weak coupling phase (and thus of the nonuniversality with KPZ) is that, for small $p_{1}$, the suppression of peaks and spikes in the interface is stronger than what can be described by the standard diffusion term in KPZ. Phenomenologically, this might be described by a generalization of KPZ where a lower power power of the Laplacian is added, e.g., in Fourier space $\partial_{t} h=-v^{\prime} k^{\mu} h-v k^{2} h+\frac{1}{2} \lambda(\mathbf{k} h)^{2}+\eta$ with $v^{\prime}>0$ and $0<\mu<2[44,45]$.

Since the present work is mainly numerical, and moreover based only on one particular model, the most obvious open problems are to support our claims with mathematical arguments and to do simulations for more general model classes. Apart from that, there are several minor open problems, such as the behavior near the tricritical point in $D=3$. Also, there are several problems we have not yet mentioned. For instance, at the sponge transition for bond percolation, a supercritical cluster with $p>p_{c}$ coexists with a critical cluster with $p=1$. A more general problem would be to find the region(s) in control parameter space where one giant cluster with $p=p_{1}$ coexists with another one with $p=p_{2}$.

Finally, I should mention theories like the quenched KPZ (qKPZ) model [3,46-50]. This model defines indeed a universality class distinct from the ordinary KPZ model, so it would seem to contradict our claim that folklore lumps models with and without quenched randomness into the same universality class. But there are two reasons why this is not true. On the one hand, it seems that qKPZ defines its own universality class only at and near the depinning transition 
(which, of course, does not exist for KPZ). As was stressed in the introduction, we should make a clear distinction between the depinning transition and propagating interfaces at finite velocities, which is the subject of the present paper. The other reason is that, to my knowledge, the qKPZ class has been discussed only in the absence of overhangs. One of the main messages of the present paper is that, even though overhangs can be neglected in its absence, they are essential for understanding moving interfaces in the presence of quenched disorder.

\section{ACKNOWLEDGMENTS}

I thank Michael Damron for very helpful correspondence and Joachim Krug, Wolfhard Janke, Werner Krauth, and Gerhard Gompper for discussions. I also want to thank Maya Paczuski and Golnoosh Bizhani for collaborations during very early step of this work and the Complexity Science Group at the University of Calgary as well as the Max-Planck Institute for the Physics of Complex Systems, Dresden, for generous grants of computer time.
[1] M. Kardar, G. Parisi, and Y.-C. Zhang, Dynamic Scaling of Growing Interfaces, Phys. Rev. Lett. 56, 889 (1986).

[2] P. Meakin, The growth of rough surfaces and interfaces, Phys. Rep. 235, 189 (1993).

[3] A.-L. Barabási and H. E- Stanley, Fractal Concepts in Surface Growth (Cambridge University Press, Cambridge, UK, 2009).

[4] F. Family and T. Vicsek, Dynamics of Fractal Surfaces (World Scientific, Singapore, 1991).

[5] T. Vicsek, Fractal Growth Phenomena (World Scientific, Singapore, 1992).

[6] M. Eden, A Two-Dimensional Growth Process (University of California Press, Berkeĺey, 1961)

[7] R. Hegger and P. Grassberger, Is Diffusion Limited Aggregation Locally Isotropic or Self-Affine? Phys. Rev. Lett. 73, 1672 (1994).

[8] C.-H. Lam, H. Kaufman, and B. B. Mandelbrot, Orientation of particle attachment and local isotropy in diffusion limited aggregation (DLA), J. Phys. A: Math. Gen. 28, L213 (1995).

[9] B. Drossel and K. Dahmen, Depinning of a domain wall in the 2d random-field Ising model, Eur. Phys. J. B 3, 485 (1998).

[10] P. Grassberger, Universality of Critically Pinned Interfaces in Two-Dimensional Isotropic Random Media, Phys. Rev. Lett. 120, 200605 (2018).

[11] H. Ji and M. O. Robbins, Percolative, self-affine, and faceted domain growth in random three-dimensional magnets, Phys. Rev. B 46, 14519 (1992).

[12] B. Koiller and M. O. Robbins, Morphology transitions in threedimensional domain growth with Gaussian random fields, Phys. Rev. B 62, 5771 (2000).

[13] H.-K. Janssen, M. Müller, and O. Stenull, Generalized epidemic process and tricritical dynamic percolation, Phys. Rev. E 70, 026114 (2004).

[14] G. Bizhani, M. Paczuski, and P. Grassberger, Discontinuous percolation transitions in epidemic processes, surface depinning in random media, and Hamiltonian random graphs, Phys. Rev. E 86, 011128 (2012).

[15] P. Grassberger (unpublished).

[16] M. A. Rubio, C. A. Edwards, A. Dougherty, and J. P. Gollub, Self-Affine Fractal Interfaces from Immiscible Displacement in Porous Media, Phys. Rev. Lett. 63, 1685 (1989).

[17] V. K. Horváth, F. Family, and T. Vicsek, Dynamic scaling of the interface in two-phase viscous flows in porous media, J. Phys. A: Math. Gen. 24, L25 (1991).

[18] S. He, G. L. M. K. S. Kahanda, and P.-Z. Wong, Roughness of Wetting Fluid Invasion Fronts in Porous Media, Phys. Rev. Lett. 69, 3731 (1992).
[19] T. Halpin-Healy and Y.-C. Zhang, Kinetic roughening phenomena, stochastic growth, directed polymers and all that: Aspects of multidisciplinary statistical mechanics, Phys. Rep. 254, 215 (1995).

[20] R. Bruinsma and G. Aeppli, Interface Motion and Nonequilibrium Properties of the Random-Field Ising Model, Phys. Rev. Lett. 52, 1547 (1984).

[21] D. A. Huse and C. L. Henley, Pinning and Roughening of Domain Walls in Ising Systems Due to Random Impurities, Phys. Rev. Lett. 54, 2708 (1985).

[22] T. Nattermann and L.-H. Tang, Kinetic surface roughening. I. The Kardar-Parisi-Zhang equation in the weak-coupling regime, Phys. Rev. A 45, 7156 (1992).

[23] D. Stauffer and A. Aharony, Introduction to Percolation Theory (Taylor \& Francis, London, 1994).

[24] Actually, most simulations were done on tilted simple (hyper) cubic lattices, such that the $z$ axis is parallel to one of the space diagonals. In that case, all neighbors of a point $(\ldots, z)$ have $z^{\prime}=z \pm 1$.

[25] B. Bock, M. Damron, C. M. Newman, and V. Sidoravicius, Percolation of finite clusters and existence of infinite shielded paths, J. Stat. Phys. 179, 789 (2020).

[26] M. Damron (private communication).

[27] Actually we used, for easier coding and higher speed, helical boundary conditions on lattices with $2^{k}$ sites, such that $L \approx$ $2^{k / D}$. The difference with strictly periodic boundary conditions is negligible.

[28] H. Hu, R. M. Ziff, and Y. Deng, No-Enclave Percolation Corresponds to Holes in the Cluster Backbone, Phys. Rev. Lett. 117, 185701 (2016).

[29] J. Wang, Z. Zhou, Q. Liu, T. M. Garoni, and Y. Deng, Highprecision Monte Carlo study of directed percolation in $(d+1)$ dimensions, Phys. Rev. E 88, 042102 (2013).

[30] J. Krug and H. Spohn, Kinetic roughening of growing surfaces, in Solids Far From Equilibrium: Growth, Morphology, and Defects, edited by C. Godrèche (Cambridge University Press, Cambridge, UK, 1991).

[31] J. Kertész and D. E. Wolf, Anomalous Roughening in Growth Processes, Phys. Rev. Lett. 62, 2571 (1989).

[32] J. Krug, J. Kertész, and D. E. Wolf, Growth shapes and directed percolation, Europhys. Lett. 12, 113 (1990).

[33] B. M. Forrest and L.-H. Tang, Hypercube stacking: A PottsSpin model for surface growth, J. Stat. Phys. 60, 181 (1990).

[34] L. Roters, S. Lübeck, and K. D. Usadel, The depinning transition of a driven interface in the random-field Ising model 
around the upper critical dimension, Phys. Rev. E 66, 026127 (2002).

[35] A. Pagnani and G. Parisi, Numerical estimate of the KardarParisi-Zhang universality class in $(2+1)$ dimensions, Phys. Rev. E 92, 010101(R) (2015).

[36] P. Grassberger, Tricritical directed percolation in 2+1 dimensions, J. Stat. Mech. (2006) P01004.

[37] D. Roux, C. Coulon, and M. E. Cates, Sponge phases in surfactant solutions, J. Phys. Chem. 96, 4174 (1992).

[38] T. J. Newman and M. R. Swift, Nonuniversal Exponents in Interface Growth, Phys. Rev. Lett. 79, 2261 (1997).

[39] G. Tripathy, A. Rocco, J. Casademunt, and W. van Saarloos, Universality Class of Fluctuating Pulled Fronts, Phys. Rev. Lett. 86, 5215 (2001).

[40] D. E. Wolf, Kinetic Roughening of Vicinal Surfaces, Phys. Rev. Lett. 67, 1783 (1991).

[41] J. G. Amar and F. Family, Phase Transition in a Restricted Solid-On-Solid Surface-Growth Model in 2+1 Dimensions, Phys. Rev. Lett. 64, 543 (1990).

[42] D. A. Huse, J. G. Amar, and F. Family, Relationship between a generalized restricted solid-on-solid growth model and a continuum equation for interface growth, Phys. Rev. A 41, 7075(R) (1990).

[43] J. Krug and H. Spohn, Mechanism for Rough-To-Rough Transitions in Surface Growth, Phys. Rev. Lett. 64, 2332 (1990).

[44] M. Nicoli, R. Cuerno, and M. Castro, Unstable Nonlocal Interface Dynamics, Phys. Rev. Lett. 102, 256102 (2009).

[45] M. Nicoli, R. Cuerno, and M. Castro, Dimensional fragility of the Kardar-Parisi-Zhang universality class, J. Stat. Mech. (2013) P11001.

[46] L.-H. Tang and H. Leschhorn, Pinning by directed percolation, Phys. Rev. A 45, R8309(R) (1992).

[47] Z. Olami, I. Procaccia, and R. Zeitak, Interface roughening in systems with quenched disorder, Phys. Rev. E 52, 3402 (1995).

[48] A. Diaz-Sanchez, L. A. Braunstein, and R. C. Buceta, Does the quenched Kardar-Parisi-Zhang equation describe the directed percolation depinning models? arXiv:cond-mat/9906363.

[49] G. Ódor, Universality classes in nonequilibrium lattice systems, Rev. Mod. Phys. 76, 663 (2004).

[50] S. Atis, A. K. Dubey, D. Salin, L. Talon, P. le Doussal, and K. Wiese, Three universality Classes for Reaction Fronts in Disordered Flows, Phys. Rev. Lett. 114, 234502 (2015). 